\title{
The frequency resolved frozen phonon method for vibrational STEM-EELS
}

\author{
Paul Zeiger and Jan Rusz
}

Uppsala University, Uppsala, Sweden

The energy resolution of electron energy loss spectroscopy (EELS) has recently been reduced to a few $\mathrm{meV}$ [1]. This enables spectroscopy of the characteristic energies of vibrational excitations in matter, also known as phonons in the case of crystals.

A number of exciting high resolution EELS experiments has since been carried out, especially, on graphene-type mono- and multilayers. Both off- and on-axis EELS were shown to exhibit atomic scale changes of the vibrational EELS spectrum [2,3], enabling effectively the study of vibrational characteristics at the atomic column level. Most recently the extreme in terms of spatial resolution was achieved by obtaining measurable changes in the vibrational EELS stemming from a single Silicon atom embedded in a host matrix of monolayer graphene [4].

However, EELS in the (scanning) transmission electron microscope ((S)TEM) does not only give access to spatially resolved spectra, but by introducing a suitable aperture in the diffraction plane, one can also get access to momentum-resolved spectral information. Recently such experiments were carried out to map the phonon dispersion of hexagonal boron nitride $(\mathrm{hBN})$, monolayer $\mathrm{hBN}$ and graphene from areas of nanometer scale [5,6]. A new generation of detectors with much reduced noise levels allows to capture angle resolved vibrational EELS spectra in parallel [7], enabling efficient and quick access to momentum resolved vibrational EELS.

From a theoretical and computational point of view EELS spectra are predominantly treated within a firstorder Born approximation of the scattering process [4,5,6,8]. Another approach by Dwyer considers explicitly the strong elastic interaction of the electron beam with the specimen in a inelastic multislice scheme [9]. These approaches have in common, that explicit knowledge of the vibrational states of the specimen, the phonon modes, is required, which is often supplied by density functional theory (DFT) phonon calculations. For large systems, such as systems with an extended defect, the necessary phonon calculations can quickly become computationally prohibitively expensive.

We have recently introduced a versatile and efficient model for vibrational STEM-EELS [10], which we shall call "frequency-resolved frozen phonon multislice method" (FRFPMS). The essence of the method is, that a colored-noise thermostat based on the generalized Langevin equation is used to excite a nonequilibrium distribution of velocities in a molecular dynamics (MD) simulations in such a way, that modes, whose frequency lies within a narrow interval, are heated, while most other modes are kept at a much lower temperature [11,12,13]. For each chosen frequency, or energy bin as we call it, a MD trajectory is generated and snapshots of the atomic positions are saved regularly. Similar to the classical frozen phonon multislice method [14], these snapshots are then separately fed into elastic multislice simulations and we generate in this way one the electron beam exit wave function for each snapshot. We then extract the thermal diffuse scattering (TDS) from coherent and incoherent averages of the exit wave function. We take this TDS intensity to be the vibrational EELS signal corresponding to the frequency excited in the MD simulation. Our method does not require explicit knowledge of the phonon modes of the specimen, making it ideally suited for large systems containing thousands of atoms.

In this contribution, we are giving additional insight on some of the properties of our method. We show how the very narrow $\delta$-thermostat [11] used in our earlier work [6] can lead to difficulties in the interpretability of spectra and how to remedy it using a so-called hotspot thermostat [13]. Furthermore, 
we will show using plane wave illumination, how our method encodes momentum resolved spectral information. In Figure 1, we show how the PDOS of hBN is well reproduced in the TDS intensity in energy-bin resolved diffraction patterns. Note that such diffraction patterns are currently not measurable, but they visualize nicely how the signal is distributed in the diffraction plane as a function of energy bin. In Figure 2, we display spectra at selected positions in the diffraction plane in Figure 2. Note the spectral differences at symmetrically equivalent positions in the diffraction plane.

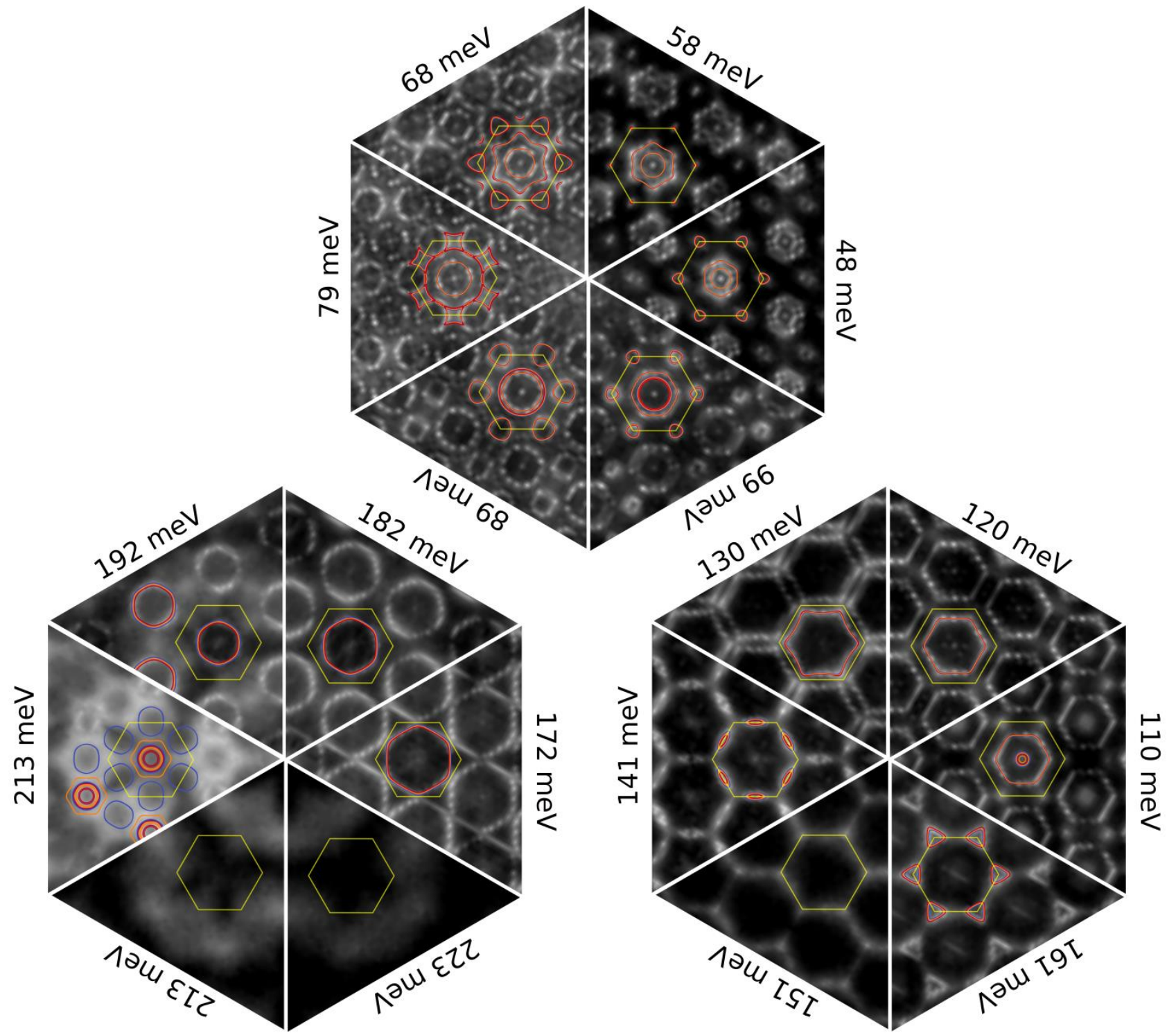

Figure 1. Figure 1: Pizza plots - comparison of energy bin resolved TDS intensity in the diffraction plane with c-axis projection of the phonon dispersion of $\mathrm{hBN}$. The EELS intensity is shown in logarithmic grayscale with black for the lowest values and white for the largest values. The projection of the 3-d phonon dispersion along the c-axis of $\mathrm{hBN}$ is overlaid. The yellow hexagon indicates the outline of one unit cell in reciprocal space. Each of the inward facing tips of the equilateral triangles cut out of the diffraction patterns is located at the (000) position in the diffraction pattern. The structure model and MD potential used for this figure is the same as in Ref. [10], but here parallel illumination was considered instead of a convergent beam. 


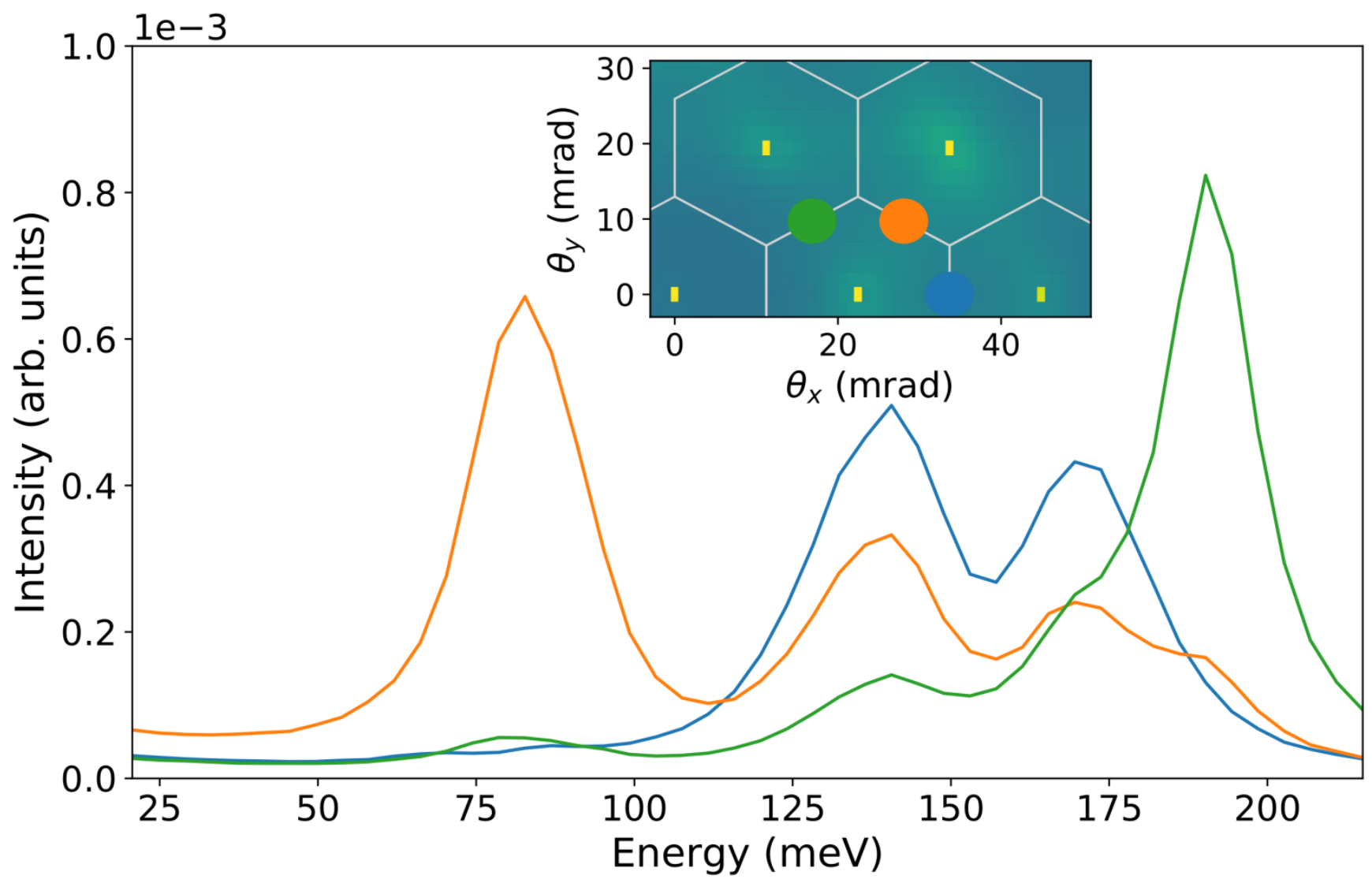

Figure 2. Figure 2: Comparison of spectra at different points in the diffraction plane. In the inset, the position and size of the detector ( 3 mrad collection semi-angle) corresponding to the similarly colored line in the plot is shown. The center of the diffraction pattern (direct beam) is located at the $(0,0) \mathrm{mrad}$ position in the diffraction pattern. The yellow hexagons indicate the Brillouin zone boundaries.

References

[1] O. L. Krivanek, T. C. Lovejoy, N. Dellby, T. Aoki, R. W. Carpenter, P. Rez, E. Soignard, J. Zhu, P. E. Batson, M. J. Lagos, R. F. Egerton, P. A. Crozier, Nature 514, 209 (2014)

[2] F. S. Hage, D. M. Kepaptsoglou, Q. M. Ramasse, L. J. Allen, Physical Review Letters 122, 016103 (2019)

[3] K. Venkatraman, B. D. A. Levin, K. March, P. Rez, P. A. Crozier. Nature Physics 15, 1237 (2019)

[4] F. S. Hage, G. Radtke, D. M. Kepaptsoglou, M. Lazzeri, Q. M. Ramasse, Science 367, 1124 (2020)

[5] F. S. Hage, R. J. Nicholls, J. R. Yates, D. G. McCulloch, T. C. Lovejoy, N. Dellby, O. L. Krivanek, K. Refson, Q. M. Ramasse, Science Advances 4:eaar7495 (2018)

[6] R. Senga, K. Suenaga, P. Barone, S. Morishita, F. Mauri, T. Pichler, Nature 573, 247 (2019)

[7] B. Plotkin-Swing, G. J. Corbin, S. De Carlo, N. Dellby, C. Hoermann, M.V. Hoffman, T. C. Lovejoy, C. E. Meyer, A. Mittelberger, R. Pantelic, L. Piazza, O. L. Krivanek, Ultramicroscopy 217, 113067 (2020) [8] R. J. Nicholls, F. S. Hage, D. G. McCulloch, Q. M. Ramasse, K. Refson, J. R. Yates, Physical Review B 99, 094105 (2019)

[9] C. Dwyer, Physical Review B 96, 224102 (2017)

[10] P. M. Zeiger, J. Rusz, Physical Review Letters 124, 025501 (2020)

[11] M. Ceriotti, G. Bussi, M. Parrinello, Journal of Chemical Theory and Computation 6, 1170 (2010) [12] M. Ceriotti, M. Parrinello, Procedia Computer Science 1, 1607 (2010) 
[13] R. Dettori, M. Ceriotti, J. Hunger, C. Melis, L. Colombo, D. Donadio, Journal of Chemical Theory and Computation 13, 1284 (2017)

[14] R. F. Loane, P. Xu, J. Silcox, Acta Crystallographica Section A: Foundations of Crystallography 47, 267 